Epidemiology and Infection

cambridge.org/hyg

\section{Original Paper}

Cite this article: Varghese BM, Dent E, Chilver M, Cameron S, Stocks NP (2018). Epidemiology of viral respiratory infections in Australian working-age adults (20-64 years): 2010-2013. Epidemiology and Infection 146, 619-626. https://doi.org/10.1017/S0950268818000286

Received: 28 July 2017

Revised: 3 January 2018

Accepted: 24 January 2018

First published online: 21 February 2018

\section{Key words:}

Influenza; respiratory infections; surveillance; virus infection

Author for correspondence:

N. P. Stocks, E-mail: nigel.stocks@adelaide. edu.au

\title{
Epidemiology of viral respiratory infections in Australian working-age adults (20-64 years): 2010-2013
}

\section{B. M. Varghese ${ }^{1}$, E. Dent ${ }^{1,2}$, M. Chilver ${ }^{3}$, S. Cameron ${ }^{1}$ and N. P. Stocks ${ }^{3}$}

${ }^{1}$ School of Public Health, The University of Adelaide, Adelaide 5000, South Australia, Australia; ${ }^{2}$ Torrens University Australia, 220 Victoria Square Adelaide, South Australia, Baker Heart and Diabetes Institute, Victoria, Australia and ${ }^{3}$ Australian Sentinal Practices Research Network (ASPREN), Discipline of General Practice, School of Medicine, The University of Adelaide, Adelaide 5000, South Australia, Australia

\section{Abstract}

Acute respiratory infections cause significant morbidity and mortality accounting for 5.8 million deaths worldwide. In Australia, influenza-like illness (ILI), defined as cough, fever and fatigue is a common presentation in general practice and results in reduced productivity and lost working days. Little is known about the epidemiology of ILI in working-age adults. Using data from the ASPREN influenza surveillance network in Australia (2010-2013) we found that working-age adults made up $45.2 \%$ of all ILI notifications with $55 \%$ of samples positive for at least one respiratory virus. Viruses most commonly detected in our study included influenza A (20.6\%), rhinovirus (18.6\%), influenza B (6.2\%), human meta-pneumovirus $(3.4 \%)$, respiratory syncytial virus $(3.1 \%)$, para-influenza virus $(2.6 \%)$ and adenovirus (1.3\%). We also demonstrated that influenza $\mathrm{A}$ is the predominant virus that increases ILI (by $1.2 \%$ per month for every positive influenza A case) in working-age adults during autumn-winter months while other viruses are active throughout the year. Understanding the epidemiology of viral respiratory infections through a year will help clinicians make informed decisions about testing, antibiotic and antiviral prescribing and when the beginning of the 'flu season' can be more confidently predicted.

\section{Introduction}

Acute respiratory infections cause significant morbidity and mortality accounting for 5.8 million deaths worldwide in 2010 [1,2]. In Australia, influenza-like illness (ILI), defined as cough, fever and fatigue is a common presentation in general practice and results in reduced productivity and lost working days [3]. The known viral or bacterial pathogen is established in only $50-60 \%$ of ILI cases while the rest are usually of unknown aetiology. Of those with a known cause, $50 \%$ are due to influenza while the remaining are due to a respiratory syncytial virus (RSV), para-influenza virus (PIV), adenovirus (ADV), rhinovirus (HRV), human metapenumovirus (HMPV) and others [4].

Working-age adults constitute a significant proportion of the population in developed countries and are generally considered to be at low risk of complications from ILIs, and in turn, have generally been overlooked. However, working-age adults can also suffer from considerable morbidity as shown by the influenza pandemic of 1919, 1967 and $2009[5,6]$. Although infection is often self-limited in this group, during a pandemic an economic loss of $\$ 60$ billion USD per year could be expected globally [7]. Additionally, on an individual basis, an average of 4-6 working days may be lost per infection/case [8].

At any given point in time, there are many respiratory pathogens other than influenza circulating within the community. However, although influenza viruses have received justified attention due to their ability to cause pandemics, the co-circulation of non-influenza viruses (NIVs) such as rhinovirus during the $2009 \mathrm{H} 1 \mathrm{N1}$ pandemic and in later 'flu seasons', have highlighted the need to monitor NIVs in conjunction with influenza surveillance $[9,10]$.

The aim of this study was to describe the viral aetiology and epidemiology of ILI in Australian working-age adults.

\section{Methods}

\section{ILI cases in general practice}

The Royal Australian College of General Practitioners (RACGP) established the Australian Sentinel Practices Research Network (ASPREN) as a national infectious disease surveillance system for Australia in 1991 [11]. ASPREN's main purpose was to monitor infectious diseases that were commonly seen in general practice, including influenza, and to allow for the 
development of early prevention strategies in the event of a pandemic. Data reporting became web-based in 2006, with automated de-identified data extraction from patient management software being introduced in 2010. Apart from ILI, other conditions such as gastroenteritis, chicken pox and shingles were also reported by more than 250 General Practitioners (GPs) across Australia. However, the focus of this paper is ILI. Participating GPs were recruited with a target of one GP per 200000 population for metropolitan areas and one GP per 50000 population in rural and remote locations [3]. Data were collected through weekly de-identified data extraction from medical records, webbased reporting and faxed paper forms [11, 12].

\section{Specimen collection and testing}

Swab testing was introduced in May 2010 and ASPREN GPs were asked to systematically collect nasopharyngeal or nasal swabs from approximately $25 \%$ of patients presenting with ILI. Specimens were then forwarded to the Institute of Medical and Veterinary Science (IMVS) where real-time reverse transcription polymerase chain reaction (RT-PCR) tests were used to identify influenza (IFA and IFB) and NIVs (HRV, ADV, PIV, HMPV and RSV) [3]. Western Australian swab data were excluded due to GPs using a different swab-testing system.

\section{Analysis}

Weekly ILI rates were calculated for the study period defined as consultations and ILI cases notified to ASPREN between 1 January 2010 and 31 December 2013. ILI rates were calculated using the formula:

$$
\text { ILI rate }=\frac{\text { Number of ILI cases }}{\text { Total GP consultations }} \times 1000
$$

Frequencies were used for descriptive analysis and crosstabulations of the different variables in the swab dataset. The proportions of swabs testing positive for influenza and NIVs were calculated by week and month of the year. Chi-square $\left(\chi^{2}\right)$ tests were used to examine whether virus detection was associated with differen demographic characteristics. Logistic regression was used for comparison across age groups (children 0-19 years; younger working adults 20-49 years; older working adults 50-64 years; and older adults $>65$ years), sex, state and month of specimen collection. All statistical tests were two-sided with $\alpha=$ 0.05 for statistical significance. Analyses were undertaken using IBM SPSS (v.20, IBM Corp, Armonk, NY, USA) and Stata software (v.13, StataCorp LP, Texas, USA).

Bivariate Poisson regression models with identity link were initially used to model the monthly contribution of each of the respiratory viruses to ILI consultations among working-age adults. However, as overdispersion was apparent in the fit of these models, negative binomial regression models (NBR) were instead used [13]. A multivariable NBR was then fit, with the criterion for inclusion in the initial multivariable model respiratory viruses with $P<0.2$ in the bivariate models. Viruses that were not significant in the multivariable model at $P<0.05$ were then removed, resulting in the final NBR model.

\section{Ethical statement}

Data provided by GPs to ASPREN are de-identified and used for public health surveillance of ILI in Australia; therefore, there was no requirement for Human Research Ethics Committee approval. However, informed written consent was obtained from patients for the collection of swab samples and use of de-identified results for surveillance purposes.

\section{Results}

\section{ILI surveillance}

There were 2901788 reported consultations made at ASPREN practices between 1 January 2010 and 31 December 2013. Of these, 22429 cases met the ILI case definition: females - 11679 (52\%) and males - 10746 (48\%) with an overall ILI rate of 7.7 cases per 1000 consultations. Working-age adults (20-64 years) represented $45 \%$ of ILI cases (data not shown).

The age-stratification analysis shows that working-age adults were not overrepresented in either the ILI notifications or the swab dataset (Fig. 1).

Over the 4 years of surveillance, 2012 had higher ILI rates than other years (12 cases/1000) followed by 2011 (nine cases/1000), 2010 (8.5 cases/1000) and the lowest rate was seen in 2013 (5.5 cases/1000).

\section{Virus detection}

Among the 22429 ILI cases, 5031 (22.0\%) swab samples were taken and tested for influenza and other respiratory viruses. Of these

\section{ASPREN Age Stratification Unswabbed ILI Patients}
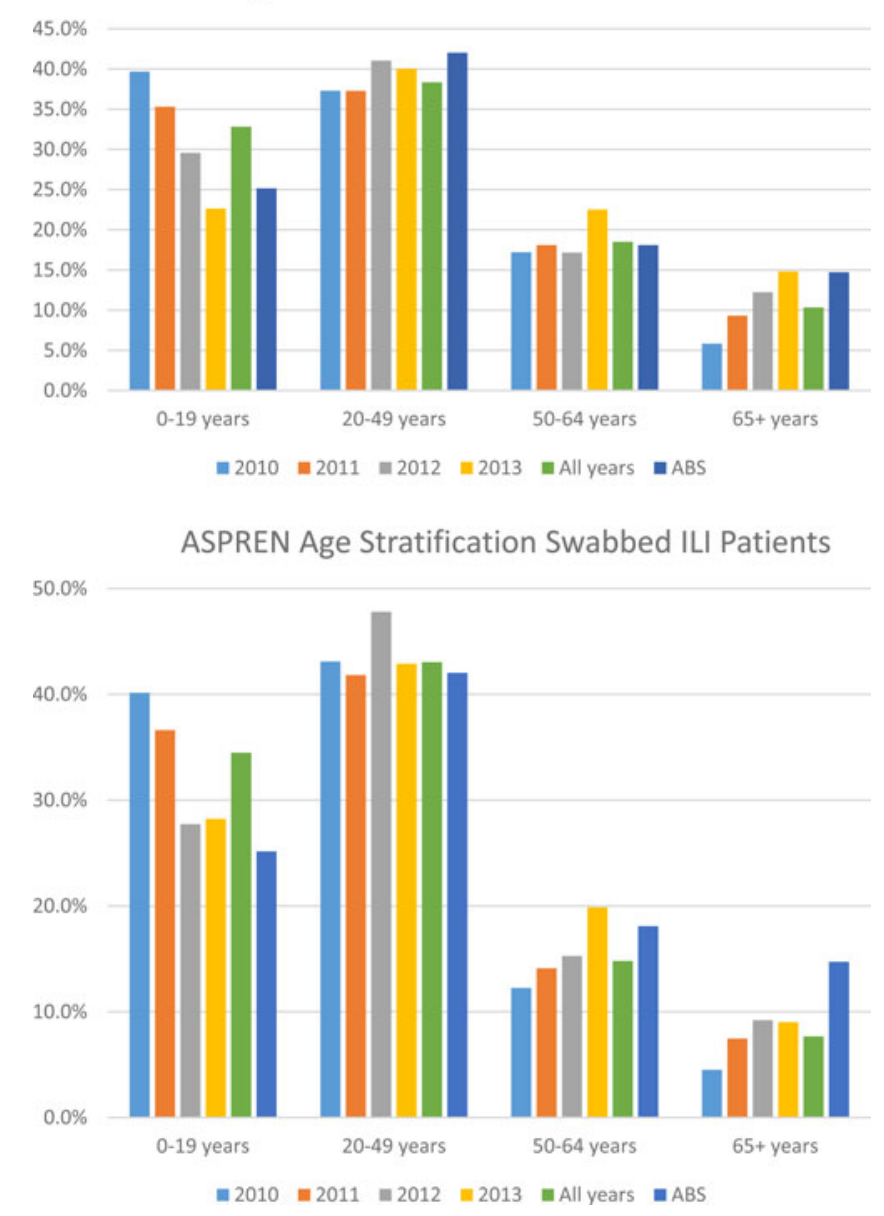

Fig. 1. Percentage of unswabbed and swabbed of ASPREN ILI patients by age group compared to Australian Bureau of Statistics (ABS) data: 2010-2013. Source: ABS (2014). Population by Age and Sex, Regions of Australia. 
samples, 3201 (32.0\%) were from individuals of working-age (2064) and 1745 (54.0\%) were female. In the study population, there were 1789 (55.8\%) cases positive for at least one virus and a single infection was identified in 1677 (52.4\%) patients. Influenza A and rhinovirus were most commonly detected, with an incidence proportion of $20.6 \%(661 / 3201)$ and $18.6 \%$ (596/3201), respectively. Of the 860 samples examined that were positive for influenza, $77.0 \%$ were type A and $23 \%$ were type B. Other viruses were found in $<5.0 \%$ of the samples. The presence of mycoplasma pneumonia was detected in 41 cases, of which the majority (30 cases) were in working-age adults.

The age distribution of cases positive for respiratory viruses is summarised in Table 1. The proportion of ILI patients positive for IFA among those aged 20-49 years was slightly lower than those aged 50-64 years and considerably higher than those aged $65+(\mathrm{OR}=1.42,95 \% \mathrm{CI}: 1.12-1.79) . \mathrm{H} 1 \mathrm{~N} 1$ (2009) proportions were higher in younger adults than in older working adults and the elderly ( $\mathrm{OR}=4.85,95 \% \mathrm{CI}$ : 2.41-9.75). Viruses such as RSV, $\mathrm{ADV}$ and PIV3 was more common in younger age groups $(<20$ years; $6 \%, 3.3 \%$ and $4.4 \%$, respectively) than those in the working and older age groups $(P<0.05)$. Within the working-age groups, older adults (50-64 years) had significant higher RSV (5.1\%) and PIV (5.1\%) cases than younger adults (20-49 years, $2.4 \%$ and $1.7 \%$, respectively; $P<0.05)$.

Within the working-age groups, co-infections were seen in 112 patients (3.4\% of swabbed ILI cases). The most frequent combinations were IFA + HMPV $(n=16)$, IFA + HRV $(n=13)$, HMPV + $\operatorname{HRV}(n=13), \operatorname{IFB}+\operatorname{HMPV}(n=9), \operatorname{ADV}+\operatorname{HRV}(n=6)$ and IFB $+\mathrm{HRV}(n=6)$. The aetiological distribution of ILI co-infections by age group is shown in Table 2. It was also observed that younger adults recorded more co-infections than older working-age adults ( $34 \%$ and $14 \%$, respectively). Additionally, co-infections of two or more viruses were detected in four individuals, three of which were aged $0-5$ years.

\section{Contribution of respiratory viruses to ILI}

Negative binomial regression

The results of the final NBR model of respiratory viruses on ILI consultations showed that ILI rates in working-age adults (2064 years) increases by $1.2 \%$ [95\% CI: $1.0049-1.0185$ ] per month for every positive IFA result.

\section{Seasonal distribution}

Figure 2 provides a snapshot of the ILI activity and various respiratory viruses that circulated within the study population by a week from 2010 to 2013 . Overall, there was a consistent pattern for ILI activity starting from a baseline level at the beginning of the year (weeks 1-8), with a slight rise during the autumn months of April-May and then increasing during the winter months of June-September. A peak is reached in August and ILI activity returns to baseline during the summer month of December. However, the ILI activity peak occurred very late in the spring months of October in 2010 (week 40) while in 2011 and 2013 the peak was during mid-August (week 33). Further, ILI rates peaked much earlier in 2012 (week 29; 26 cases/1000) than the peak in 2011 and 2013, with the ILI peak being highest in this year.

In Australia, 2012 was the most severe 'flu season' between 2010 and 2013, characterised as an early season dominated by $\mathrm{H} 3 \mathrm{~N} 2$, which is associated with more severe illness in the young and elderly [14]. ASPREN data support these findings.
The proportion or number of samples positive for influenza in 2012 was $33.9 \%$ (380) compared with $23.1 \%$ (480) in other years. Influenza A was the dominant type with $85.5 \%$ of cases positive for influenza A. Additionally, overall virus detection was higher at $57.9 \%$.

Influenza viruses circulated throughout the year, but increased activity was seen during April-October. IFA and IFB often co-circulated throughout the study period but peaked at different times annually (IFA usually peaks in July and IFB usually peaks in August). HRV was active throughout the year peaking in February-March and continuing to be the dominant NIV in the following months until the peak of influenza viruses and further rising to a peak in the spring and early summer (Fig. 3). PIV3 was detected in high numbers in the spring months with December seeing a decrease in other respiratory viruses. RSV followed the HRV pattern of greater activity before and after the IFA peak. Clear cut seasonality was not seen for ADV and PIV 1,2 as they circulated sporadically throughout the study period.

The proportions of influenza and NIV's varied from year to year with 2010 and 2013 seeing a higher proportion of NIV (29.5\%, $33.9 \%$ ) compared with influenza virus $(21.3 \%, 19.5 \%)$ (Fig. 3 ).

\section{Influenza vaccination}

Influenza vaccination amongst swabbed ILI cases was $20.3 \%$ overall, varying from 15\% in 2010 to $24 \%$ in 2013 (see for Fig. S1).

\section{Discussion}

This is the first Australian study to report the viral aetiology and epidemiology of ILI in working-age adults using representative community surveillance data. Working-age adults made up $45.2 \%$ of all ILI notifications with $56 \%$ of samples positive for at least one respiratory virus, a finding consistent with recent international data [15-17]. In agreement with other studies [18, 19], the viruses most commonly detected in our study included IFA (20.6\%), HRV (18.6\%), IFB (6.2\%), HMPV (3.4\%), RSV (3.1\%), PIVs (3\%) and ADV (1.3\%). Further, we have demonstrated that IFA is the predominant virus that increases ILI (by $1.2 \%$ per month for every positive IFA case) in working-age adults during autumn-winter months while NIVs such as HRV, ADV and PIVs account for ILI activity throughout the year.

Rates of ILI presentations in working-age adults, compared with other groups, in our study appear to be high. Previous research has attributed high ILI notification rates in working-age adults to illness certification requirements by employers [20]. Secondly, it has been noted that following the 2009 pandemic, that risk perception concerning influenza among working adults was higher [21]. Age-stratification analyses show that our results are representative of the Australian population as a whole with the exception of a slight overrepresentation and underrepresentation in the under 19 and over 65 year age groups, respectively [22]. Two plausible reasons for this observation could be: the health-seeking behaviour of parents with children [23] and the proportion of older people living in residential facilities who access alternative care.

Consistent with previous studies [16, 24, 25], influenza viruses, accounting for $27 \%$ of ILI cases, were the most commonly detected viral cause of ILI in working-age adults. IFA in working-age adults was the most frequently detected viruses in this population $(20.6 \%$ of all ILI cases). This finding is supported by the regression model results where one positive IFA result contributed a $1.2 \%$ increase in ILI rates/month. Children and the elderly are commonly assumed to be the most impacted as these populations tend to have lower 


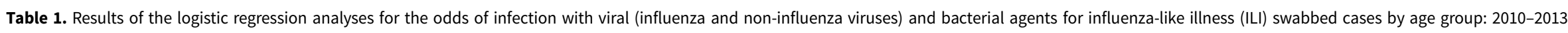

\begin{tabular}{|c|c|c|c|c|c|c|c|c|}
\hline \multirow{2}{*}{$\begin{array}{l}\text { Age group (years) } \\
\text { Respiratory agent }\end{array}$} & \multicolumn{2}{|c|}{$0-19$} & \multicolumn{2}{|c|}{$20-49$} & \multicolumn{2}{|c|}{$50-64$} & \multicolumn{2}{|c|}{$65+$} \\
\hline & $n(\%)$ & OR $(95 \% \mathrm{CL})$ & $n(\%)$ & OR $(95 \% \mathrm{CL})$ & $n(\%)$ & OR $(95 \% \mathrm{CL})$ & $n(\%)$ & OR $(95 \% \mathrm{CL})$ \\
\hline Viral agent & $999(74.1)$ & & $1317(55.4)$ & & $472(57.3)$ & & $220(49.2)$ & \\
\hline Influenza positive & $522(38.7)$ & $1.99(1.62-2.43)$ & $672(28.3)$ & $1.47(1.20-1.80)$ & $188(22.8)$ & $1.17(0.93-1.47)$ & $86(19.2)$ & ref \\
\hline Influenza A & $321(23.8)$ & $1.56(1.22-1.98)$ & $511(21.5)$ & $1.42(1.12-1.79)$ & $150(18.2)$ & $1.19(0.92-1.55)$ & $68(15.2)$ & ref \\
\hline H1N12009 & $126(9.3)$ & $5.04(2.48-10.21)$ & $206(8.6)$ & $4.85(2.41-9.75)$ & $38(4.6)$ & $2.59(1.22-5.51)$ & $8(1.8)$ & ref \\
\hline Influenza B & 201(14.9) & $3.60(2.25-5.67)$ & $161(6.7)$ & $1.67(1.04-2.69)$ & $38(4.6)$ & $1.13(0.65-1.95)$ & $18(4.02)$ & ref \\
\hline Non-influenza virus positive & $477(35.3)$ & $1.09(0.93-1.29)$ & $645(27.1)$ & $0.89(0.76-1.04)$ & $284(34.5)$ & $1.12(0.94-1.34)$ & $134(29.9)$ & ref \\
\hline Rhinovirus & $228(16.9)$ & $1.01(0.79-1.27)$ & $444(18.7)$ & $1.11(0.89-1.39)$ & $152(18.4)$ & $1.09(0.85-1.41)$ & $75(16.7)$ & ref \\
\hline RSV & $81(6)$ & $1.07(0.69-1.66)$ & $58(2.4)$ & $0.43(0.27-0.69)$ & $42(5.1)$ & $0.91(0.56-1.47)$ & $25(5.6)$ & ref \\
\hline Adenovirus & $59(4.4)$ & & $34(1.4)$ & & $8(0.9)$ & & $0(0)$ & \\
\hline Para-influenza & $64(4.7)$ & $1.28(0.75-2.20)$ & $41(1.7)$ & $0.45(0.25-0.80)$ & $42(5.1)$ & $1.44(0.82-2.54)$ & $16(3.6)$ & ref \\
\hline Human Metapneumovirus & $45(3.3)$ & $0.81(0.47-1.38)$ & $68(2.8)$ & $0.69(0.41-1.14)$ & $40(4.8)$ & $1.20(0.70-2.08)$ & $18(4.0)$ & ref \\
\hline Bacterial agent & $25(1.9)$ & $1.65(0.63-4.29)$ & $31(1.3)$ & $1.17(0.46-3.01)$ & $13(1.6)$ & $1.31(0.46-3.71)$ & $5(1.1)$ & ref \\
\hline Mycoplasma pneumoniae & $9(0.6)$ & & $21(0.8)$ & & $9(1.1)$ & & $2(0.4)$ & \\
\hline Pertussis & $16(1.2)$ & & $10(0.4)$ & & $4(0.5)$ & & $3(0.6)$ & \\
\hline Total ILI swabbed cases ( $n$ ) & 1349 & & 2377 & & 824 & & 447 & \\
\hline
\end{tabular}

Models adjusted for month of specimen collection and State. Shaded cells denote statistical significance at $P<0.05$ and blank cells indicate insufficient data. Total swabbed cases do not equal to 5031 due to missing data on age. Highlighted age group is our study population. 
Table 2. Distribution of viruses and bacteria related to co-infections among all influenza-like illness (ILI) patients by age group: 2010-2013

\begin{tabular}{|c|c|c|c|c|}
\hline Age group & 0-19 years & 20-49 years & 50-64 years & $>65$ years \\
\hline Co-infection ( $n \%$ ) & $n=104$ & $n=79$ & $n=33$ & $n=17$ \\
\hline IFA + RSV & $1(0.9)$ & $1(1.2)$ & 0 & $3(17.6)$ \\
\hline $\mathrm{IFA}+\mathrm{HRV}$ & $13(12.5)$ & $9(11.3)$ & $4(12.1)$ & $2(11.7)$ \\
\hline $\mathrm{IFA}+\mathrm{ADV}$ & $2(1.9)$ & 0 & 0 & 0 \\
\hline IFA + PIV3 & $2(1.9)$ & $1(1.2)$ & $1(3.0)$ & 0 \\
\hline $\mathrm{IFA}+\mathrm{HMPV}$ & $9(8.6)$ & $13(16.4)$ & $3(9.1)$ & $4(23.5)$ \\
\hline IFA + Mycoplasma & $2(1.9)$ & $4(5.1)$ & $1(3.0)$ & 0 \\
\hline IFA + Pertussis & $0(0)$ & $1(1.2)$ & 0 & $1(5.8)$ \\
\hline $\mathrm{IFB}+\mathrm{HRV}$ & $4(3.8)$ & $4(5.1)$ & $2(6.1)$ & 0 \\
\hline $\mathrm{IFB}+\mathrm{ADV}$ & $2(1.9)$ & $1(1.2)$ & 0 & 0 \\
\hline IFB + PIV3 & $2(1.9)$ & $1(1.2)$ & 0 & $1(5.8)$ \\
\hline IFB + HMPV & $6(5.7)$ & $7(8.8)$ & $2(6.1)$ & 0 \\
\hline IFB + Mycoplasma & $2(1.9)$ & 0 & 0 & 0 \\
\hline IFB + Pertussis & $3(2.8)$ & 0 & 0 & 0 \\
\hline RSV + HRV & $12(11.5)$ & $3(3.7)$ & $1(3.0)$ & 0 \\
\hline $\mathrm{RSV}+\mathrm{ADV}$ & $4(3.8)$ & 0 & 0 & 0 \\
\hline RSV + PIV1 & 0 & 0 & $1(3.0)$ & 0 \\
\hline RSV + PIV3 & $3(2.8)$ & 0 & 0 & 0 \\
\hline RSV + HMPV & $1(0.9)$ & $2(2.5)$ & $2(6.1)$ & $2(11.7)$ \\
\hline RSV + Mycoplasma & 0 & 0 & 0 & $1(5.8)$ \\
\hline RSV + Pertussis & 0 & $1(1.2)$ & 0 & 0 \\
\hline$A D V+H R V$ & $12(11.5)$ & $5(6.3)$ & $1(3.0)$ & 0 \\
\hline$A D V+H M P V$ & $2(1.9)$ & 0 & 0 & 0 \\
\hline ADV + PIV3 & $3(2.8)$ & 0 & 0 & 0 \\
\hline ADV + Mycoplasma & 0 & $2(2.5)$ & $1(3.0)$ & 0 \\
\hline HMPV + PIV1 & $1(0.9)$ & 0 & 0 & 0 \\
\hline HMPV + PIV2 & 0 & 0 & $1(3.0)$ & 0 \\
\hline HMPV + PIV3 & 0 & $4(5.1)$ & $1(3.0)$ & 0 \\
\hline HMPV + HRV & $6(5.7)$ & $8(10.1)$ & $5(15.1)$ & $2(11.7)$ \\
\hline HRV + PIV3 & $6(5.7)$ & $2(2.5)$ & $1(3.0)$ & 0 \\
\hline HRV + Pertussis & $2(1.9)$ & $3(3.7)$ & $1(3.0)$ & $1(5.8)$ \\
\hline HRV + Mycoplasma & $2(1.9)$ & $6(7.6)$ & $4(12.1)$ & 0 \\
\hline HRV + PIV2 & $1(0.9)$ & $1(1.2)$ & 0 & 0 \\
\hline PIV1 + Pertussis & $1(0.9)$ & 0 & 0 & 0 \\
\hline PIV3 + Pertussis & 0 & 0 & $1(3.0)$ & 0 \\
\hline
\end{tabular}

immunity or are frailer [4]. However, compared with those aged over 65 years, young adults aged 20-49 years had a higher odds of IFA infection due to H1N1pdm09 and greater absolute numbers. Studies have also reported sustained higher swab positivity, higher rates of outpatient ILI consultation and increased rates of hospitalisation, an impact which is unusual among 'young working adults' which carried over even after the pandemic years (2010, 2011 and 2013) when H1N1pdm09 was the circulating strain [26, 27]. Studies conducted during the 2009 pandemic have suggested that in comparison with younger age groups ( $<50$ years), older adults and the elderly may have benefited from pre-existing immunity from past exposure to H1N1pdm09 strains that are similar to the 1918 strain [28, 29]. Higher 'pre-existing anti-IFA antibodies' were found in elderly adults born before the 1940-1950s compared with those born after by a Finnish and Belgian study [18, 30]. This could account for the higher attack rates of H1N1pdm09 among young adults during and after the pandemic.

Even though the proportion of IFB was higher in those aged 0-19 years, which is consistent with the literature [31], they were the third most common virus among working-age adults (6.2\% of all ILI cases) with increased levels of circulation seen in the spring months of 2012 (37\% in weeks $36-48 ; 33 \%$ in weeks 49-53) and 2013 (30\% in weeks 36-48) compared with other years. This pattern of increased influenza B activity followed by high IFA activity in 2012 and 2013 has been observed in North America and Europe [32, 33].

2012 saw a moderate to severe flu season characterised by high levels of $\mathrm{H} 3 \mathrm{~N} 2$ circulation, where $70 \%$ of swabbed patients were not vaccinated, this being higher than other years. Additionally, vaccine effectiveness against medically attended ILI was estimated to be low in this year $23 \%$ (95\% CI, $-4 \%$ to $43 \%$ ) in 2012 compared with $60 \%(95 \%$ CI 45-70) in 2013 [34].

NIVs constituted $29 \%$ of all ILI cases in this population, which is in accordance with an ILI study conducted among university students [24]. Following a typical 'flu-season', ILI rates generally start to decline in spring with studies showing that a decrease in influenza virus detection coincides with an increase in NIVs [35]. The percentage of NIVs in our study is higher than in a Korean study where $23 \%$ of adult respiratory samples had other viruses in addition to influenza [36]. Positivity may vary between studies due to the differences in case definitions, viral detection methods, viruses targeted and study population characteristics [9].

When we examined the types of NIVs found in our study, HRV was predominant (18\%). Data from 12 European countries provide similar estimates in immune-competent adults at their first visit with $5 \%$ positive at follow-up [37]. Their study also found that even though viral shedding lasted between 1 and 2 weeks, re-infections were more common than prolonged infection and underlying co-morbidities could enhance the chances of re-infection [37]. The estimate of HRV from our study was similar to a Brazilian study that found $19.6 \%$ of ILI in healthy adults related to HRV infections [17]. Our study demonstrates overlapping patterns of circulation for the predominant respiratory viruses such as IFA and HRV especially in the cooler months of the year. Even though HRV circulates year-round, the noticeable feature of HRV is that its activity is greater before and after the peak of IFA. This finding is congruent with studies that have found higher HRV circulation coinciding with lower influenza virus activity [38].

RSV was detected in $3 \%$ of ILI swabs which is the same as in a Korean study [36] but lower than a prospective study done in the USA [39] that found a rate of 7\% in healthy adults aged 18-60 years. This US study also reported that influenza and RSV infections were the most common causes for work absence constituting about $66 \%$ and $38 \%$ respectively of cases among those working in universities and healthcare services [39]. Similar to HRV, RSV also has a higher potential for re-infections across all age groups and a mean duration of illness longer than influenza (9.5 vs. 6.8 days) which could add to medical costs and lost productivity [39]. Lastly, RSV was observed to circulate in the same pattern as influenza with peaks in winter months (July) consistent with what was 


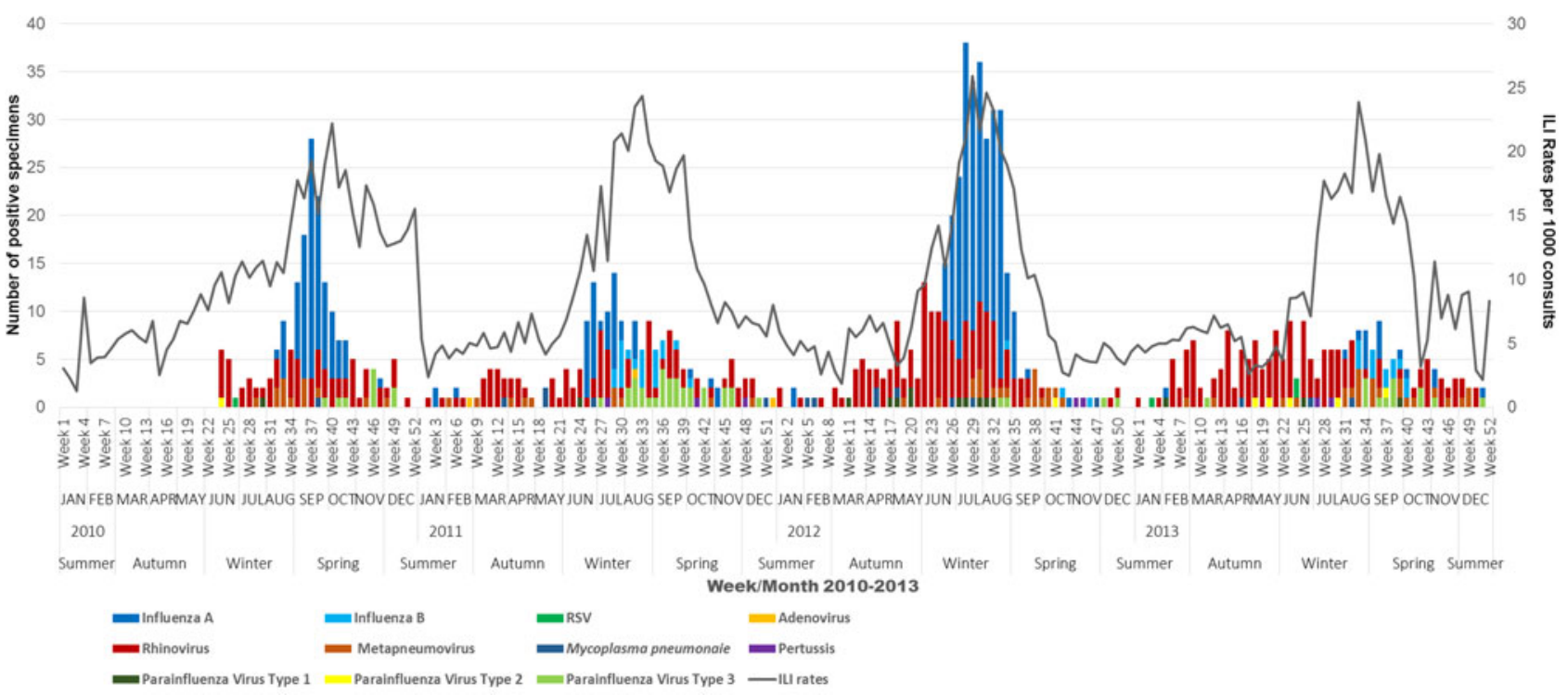

Fig. 2. Overview of ILI activity (ILI rates) and corresponding viral activity of influenza and other respiratory viruses for working-age adults by week, month and season, 2010-2013. Note: Zero virus detections until week 23 in 2010 as swab testing was only introduced in May 2010.

observed earlier, possibly due to their associations with cold temperatures in temperate regions [40].

ILI rates decline after spring and achieve baseline levels in the temperate parts of Australia during summer spanning December (weeks 49-53) until March. NIVs are the predominant cause of baseline ILI activity during spring-summer. In contrast to this usually observed pattern, the spring-summer of 2010-2011 saw high numbers of IFA cases, which was reported by Kelly et al. (2012) who found increased influenza notifications in Victoria and across Australia during this time period [41]. The delayed IFA season in 2010, that peaked in late September-early October (spring), in contrast to the normal peak in mid-late winter (August), may explain the unusual circulation of influenza. The higher circulation of rhinovirus during the winter of 2010 may have resulted in the delayed IFA activity during spring that extended into summer (December 2010-February 2011). This phenomenon referred to as 'viral interference' or interaction is where two viruses that circulate in overlapping patterns or one after the other, compete with each other in a race to dominate the season [35, 42]. Studies from France and Sweden have also reported delayed influenza activity due to viral interaction [35, 43]. Understanding such interactions and the epidemiology of viral respiratory illness through the year could be of benefit to primary care clinicians when making decisions about swab testing and deciding when the 'flu season' has started. For instance given the low rates of IFA in the summer months doctors could chose not to test most ILI cases safe in the knowledge that most would have an NIV, conversely at the peak of the 'flu season' most ILI will be due to influenza and could be presumptively managed as such without immediate recourse to testing.

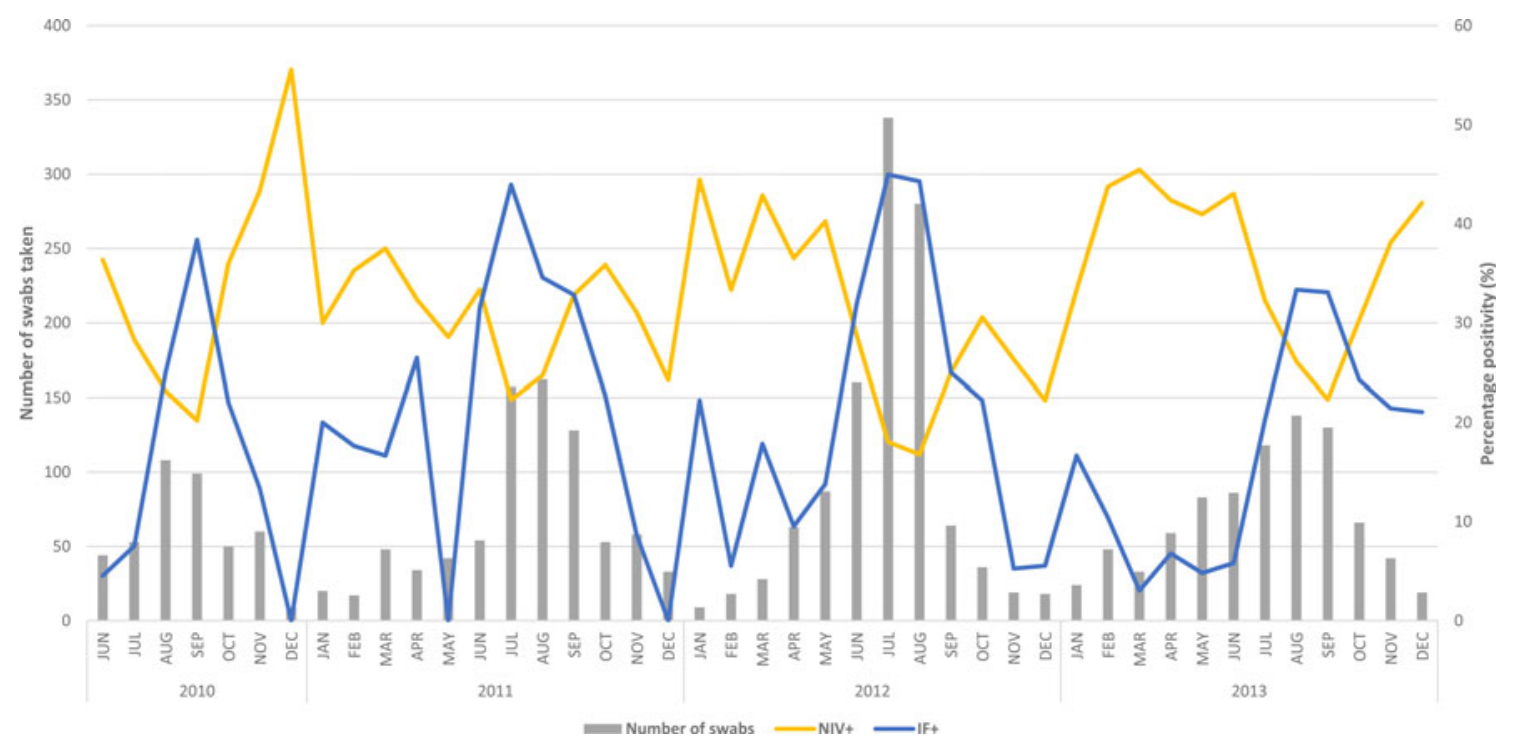

Fig. 3. Swab positivity (\%) of influenza (IF+) and non-influenza viruses (NIV+) for working-age adults by month: 2010-2013. Note: Virus positivity is only graphed from June 2010 as swab testing was only introduced in May 2010. 
Co-infections affected 112 patients or $3.4 \%$ of ILI cases, which falls within the $0.7-15.3 \%$ range found in previous studies [44]. Our finding that younger working-age adults had more co-infections than older working-age adults (with HRV being most frequently found in combination) agrees with recent research [9]. Given that the H1N1pdm09 strain constituted most of the IFA cases among working-age adults, it has been proposed in the literature that they were also at greater risk of acquiring other co-circulating viruses [9]. Further, the frequent occurrence of co-infection with HRV-Influenza combination is attributed to the nature of HRV infection that reduces the immune function by altering the normal levels of lymphocytes (high to low) and neutrophils (low to high) [45].

The present study has several limitations. Firstly, results regarding numbers of circulating viruses need to be interpreted carefully, given that estimates presented in our study were related only to those individuals presenting to their GPs and/or swabbed. Secondly, ASPREN data are limited to respiratory viruses that are currently monitored. Thirdly, date of vaccination was not recorded, therefore we are unable to ascertain whether the patients were fully vaccinated at the time of presentation (i.e. $>14$ days after vaccination received). Fourthly we could not include swab data from WA, although seasonal patterns may be different in the semi-tropical north, patterns in the more heavily populated South West are similar to the rest of Australia so the effect on the results will be small. Finally, data were not collected on work status, the purpose of GP visit and/or underlying medical conditions of patients; therefore, it was not possible to determine whether the burden of ILI-related viruses was related to co-morbidities.

Despite limitations, the ASPREN surveillance dataset has much strength. It is able to provide an improved understanding of trends over time of different respiratory viruses, their seasonality and the relative contribution of NIVs to morbidity in working-age adults. The results reinforce evidence that all 'flu's' in working-age adults are not due to influenza alone but are often caused by other respiratory viruses that produce similar symptoms. Further, understanding the seasonality patterns of respiratory viruses could improve the management of ILI in the community by reducing the use of antivirals outside the influenza peak in July/August and highlighting the viral nature of ILI throughout the year to decrease antibiotic prescribing. Continued testing is important because about $40 \%$ of adults receive antibiotics when diagnosed with ILI [46]. Possible inclusion of other recently discovered respiratory pathogens that are detected using PCR technique may be useful to explore the unknown aetiology of $40 \%$ of ILI cases [9].

Supplementary material. The supplementary material for this article can be found at https://doi.org/10.1017/S0950268818000286

Acknowledgements. The authors wish to thank all the GPs participating to the ASPREN surveillance network and would also like to acknowledge Daniel Blakely (ASPREN project officer) for providing the data, Dr Lynne Giles and Ms Suzanne Edwards for advice on statistical analysis and Ms Christina Bareja, Dr Rachel de Kluyver and Dr Sheena Sullivan for reviewing the manuscript and providing their valuable comments. The Australian Sentinel Practices Research Network is supported by the Australian Government Department of Health (the Department). The opinions expressed in this paper are those of the authors, and do not necessarily represent the views of the Department. Elsa Dent is supported by an Australian National Health and Medical Research Council Early Career Fellowship (grant no. 1112672).

Declaration of Interest. None.

\section{References}

1. Howard PF, et al. (2013) Virus detection and its association with symptoms during influenza-like illness in a sample of healthy adults enrolled in a randomised controlled vaccine trial. Influenza and Other Respiratory Viruses 7(3), 330-339.

2. Ambrosioni J, et al. (2013) Epidemiology of viral respiratory infections in a tertiary care centre in the era of molecular diagnosis, Geneva, Switzerland, 2011-2012. Clinical Microbiology and Infection 20, 0578-0584.

3. Sullivan SG, et al. (2014) Influenza vaccine effectiveness in Australia: results from the Australian sentinel practices research Network. The Medical Journal of Australia 201(2), 109-111.

4. Cedraschi C, et al. (2013) Representations of influenza and influenza-like illness in the community-a qualitative study. BMC Family Practice 14, 15. doi: 10.1186/1471-2296-14-15.

5. Reichert T, Chowell G and McCullers JA (2012) The age distribution of mortality due to influenza: pandemic and peri-pandemic. BMC Medicine 10(1), 1-15. doi: 10.1186/1741-7015-10-162.

6. Gasparini R, Amicizia D, Lai PL and Panatto D (2012) Clinical and socioeconomic impact of seasonal and pandemic influenza in adults and the elderly. Human Vaccines \& Immunotherapeutics 8(1), 21-28. doi: 10.4161/hv.8.1.17622.

7. Sands P, Mundaca-Shah C and Dzau VJ (2016) The neglected dimension of global security - a framework for countering infectious-disease crises. The New England Journal of Medicine 374, 1281-1287. doi: 10.1056/NEJMsr1600236.

8. Keech $\mathbf{M}$ and Beardsworth $\mathbf{P}$ (2008) The impact of influenza on working days lost: a review of the literature. PharmacoEconomics 26(11), 911-924.

9. Peci A, et al. (2013) Community-acquired respiratory viruses and co-infection among patients of Ontario sentinel practices, April 2009 to February 2010. Influenza and Other Respiratory Viruses 7(4), 559-566. doi: 10.1111/j.1750-2659.2012.00418.x.

10. Pascalis H, et al. (2012) Intense co-circulation of non-influenza respiratory viruses during the first wave of pandemic influenza pH1N1/2009: a cohort study in Reunion Island. PLoS ONE 7(9), e44755. doi: 10.1371/ journal.pone.0044755.

11. Parrella ADC, Pearce R and Lit JCB (2009) ASPREN surveillance system for infleunza-like illness: a comparison with FluTracking and the National Notifiable Diseases Surveillance System. Australian Family Physician 38 (11), 932-936.

12. The Australian Sentinel Practices Research Network. Become an ASPREN GP. ASPREN; 2014 [cited 2014 June 20]. Available at http:// www.aspren.com.au/Register.aspx.

13. Bollaerts K, et al. (2013) Contribution of respiratory pathogens to influenza-like illness consultations. Epidemiology and Infection 141(10), 2196-2204. doi: 10.1017/s0950268812002506.

14. Australian Government (2013) Australian Influenza Surveillance Report. Canberra: Department of Health.

15. Falchi A, et al. (2011) Nationwide surveillance of 18 respiratory viruses in patients with influenza-like illnesses: a pilot feasibility study in the French Sentinel Network. Journal of Medical Virology 83(8), 1451-1457. doi: $10.1002 / j m v .22113$.

16. Hasman H, et al. (2009) Aetiology of influenza-like illness in adults includes parainfluenzavirus type 4. Journal of Medical Microbiology 58 (Pt 4), 408-413. doi: 10.1099/jmm.0.006098-0.

17. Bellei $\mathbf{N}$, et al. (2008) Acute respiratory infection and influenza-like illness viral etiologies in Brazilian adults. Journal of Medical Virology 80(10), 1824-1827. doi: 10.1002/jmv.21295.

18. Hombrouck A, et al. (2012) Viral aetiology of influenza-like illness in Belgium during the influenza A(H1N1)2009 pandemic. European Journal of Clinical Microbiology \& Infectious Diseases 31(6), 999-1007. doi: 10.1007/s10096-011-1398-4.

19. Zimmerman RK, et al. (2014) Influenza and other respiratory virus infections in outpatients with medically attended acute respiratory infection during the 2011-12 influenza season. Influenza and Other Respiratory Viruses 8(4), 397-405. doi: 10.1111/irv.12247.

20. Miller ER, et al. (2008) Higher than expected seasonal influenza activity in Victoria, 2007. Communicable Diseases Intelligence Quarterly Report 32 (1), 63-70 
21. Troko J, et al. (2011) Is public transport a risk factor for acute respiratory infection? BMC Infectious Diseases 11, 16.

22. Australian Bureau of Statistics. 3235.0- Population by Age and Sex, Regions of Australia, 2014. Canberra: ABS; 2015 [updated 30 Nov 2017; cited $201728 \mathrm{Nov}$ ]. Available at http://www.abs.gov.au/AUSSTATS/ abs@.nsf/Lookup/3235.0Main+Features12014?OpenDocument.

23. Van Cauteren D, et al. (2012) Burden of influenza, healthcare seeking behaviour and hygiene measures during the $\mathrm{A}(\mathrm{H} 1 \mathrm{~N} 1) 2009$ pandemic in France: a population based study. BMC Public Health. 12(1), 1-8. doi: 10.1186/1471-2458-12-947.

24. Mullins J, et al. (2011) Influenza-Like illness Among university students: symptom severity and duration due to influenza virus infection compared to other etiologies. Journal of American College Health 59(4), 246-251. doi: 10.1080/07448481.2010.502197. Cited in: aph.

25. Ren L, et al. (2009) Prevalence of human respiratory viruses in adults with acute respiratory tract infections in Beijing, 2005-2007. Clinical Microbiology and Infection 15(11), 1146-1153.

26. Chan PA, et al. (2011) Distinguishing characteristics between pandemic 2009-2010 influenza A (H1N1) and other viruses in patients hospitalized with respiratory illness. PLoS ONE 6(9), e24734. doi: 10.1371/ journal.pone.0024734.

27. Ellis J, et al. (2011) Virological analysis of fatal influenza cases in the United Kingdom during the early wave of influenza in winter 2010/11. Euro Surveillance: European Communicable Disease Bulletin 16(1), 19760.

28. Skountzou I, et al. (2010) Immunity to pre-1950 H1N1 influenza viruses confers cross-protection against the pandemic swine-origin 2009 A (H1N1) influenza virus. Journal of Immunology (Baltimore, MD: 1950) 185(3), 1642-1649. doi: 10.4049/jimmunol.1000091.

29. Valkenburg SA, et al. (2011) Immunity to seasonal and pandemic influenza A viruses. Microbes and Infection/Institut Pasteur 13(5), 489-501. doi: 10.1016/j.micinf.2011.01.007.

30. Ikonen N, et al. (2010) High frequency of cross-reacting antibodies against 2009 pandemic influenza $\mathrm{A}(\mathrm{H} 1 \mathrm{~N} 1)$ virus among the elderly in Finland. Euro Surveillance: European Communicable Disease Bulletin 15(5), 19478.

31. Kelly H, Grant K, Williams S and Smith D (2009) H1n1 swine origin influenza infection in the United States and Europe in 2009 may be similar to H1N1 seasonal influenza infection in two Australian states in 2007 and 2008. Influenza and Other Respiratory Viruses 3(4), 183-188. doi: 10.1111/ j.1750-2659.2009.00088.x.

32. World Health Organization W (2012) Review of the 2011-2012 Winter Influenza Season, Northern Hemisphere. Geneva.
33. World Health Organization W (2013) Review of the 2012-2013 Winter Influenza Season, Northern Hemisphere. Geneva: WHO.

34. Sullivan SG, et al. (2016) Pooled influenza vaccine effectiveness estimates for Australia, 2012-2014. Epidemiology and Infection 144(11), 2317-2328. doi: $10.1017 / \mathrm{s} 0950268816000819$.

35. Linde A, et al. (2009) Does viral interference affect spread of influenza? Euro Surveillance: European Communicable Disease Bulletin 14(40), 19354.

36. Noh JY, et al. (2013) Laboratory surveillance of influenza-like illness in seven teaching hospitals, South Korea: 2011-2012 season. PLoS ONE 8 (5), e64295. doi: 10.1371/journal.pone.0064295.

37. Zlateva KT, et al. (2014) Prolonged shedding of rhinovirus and re-infection in adults with respiratory tract illness. The European Respiratory Journal 44(1), 169-177. doi: 10.1183/09031936.00172113.

38. Razanajatovo NH, et al. (2011) Viral etiology of influenza-like illnesses in Antananarivo, Madagascar, July 2008 to June 2009. PLoS ONE 6(3), e17579. doi: 10.1371/journal.pone.0017579.

39. Hall CB, Long CE and Schnabel KC (2001) Respiratory syncytial virus infections in previously healthy working adults. Clinical Infectious Diseases 33(6), 792-796. doi: 10.1086/322657.

40. Haynes AK, et al. (2013) Respiratory syncytial virus circulation in seven countries with global disease detection regional centers. The Journal of Infectious Diseases 208(Suppl 3), S246-S254. doi: 10.1093/infdis/jit515.

41. Australian Government. Communicable Diseases Australia(CDA) National Notifiable Diseases Surveillance System(NNDSS). Canberra: Department of Health; 2014 [24th March 2014]. Available at http:// www9.health.gov.au/cda/source/rpt_4.cfm.

42. Schnepf N, et al. (2011) High burden of non-influenza viruses in influenza-like illness in the early weeks of H1N1v epidemic in France. PLOS ONE 6(8), e23514. doi: 10.1371/journal.pone.0023514.

43. Casalegno JS, et al. (2010) Rhinoviruses delayed the circulation of the pandemic influenza A (H1N1) 2009 virus in France. Clinical Microbiology and Infection 16(4), 326-329. doi: 10.1111/j.1469-0691.2010.03167.x.

44. Yang X, et al. (2012) Etiology and clinical characteristics of influenza-like illness (ILI) in outpatients in Beijing, June 2010 to May 2011. PLoS ONE 7(1), e28786. doi: 10.1371/journal.pone.0028786

45. Xiang Z, et al. (2010) Human rhinoviruses in Chinese adults with acute respiratory tract infection. Journal of Infection 61(4), 289-298. doi: http:// dx.doi.org/10.1016/j.jinf.2010.07.001.

46. Bergus GR, Weber CA, Ernst ME and Ernst EJ (2008) Do antibiotics affect the quality of life of patients with upper respiratory tract illnesses? It might depend on one's luck. International Journal of Clinical Practice 62(6), 855-859. doi: 10.1111/j.1742-1241.2008.01775.x. 